\title{
Right Deltoid
}

National Cancer Institute

\section{Source}

National Cancer Institute. Right Deltoid. NCI Thesaurus. Code C105635.

The muscle that creates the rounded contour of the shoulder which orig inates from the lateral third of the clavicle, the superior surface of the acromion process, and the posterior border of the spine of the scapula and inserts on the lateral side of the shaft of the humerus. The deltoid muscle is innervated by the brachial plexus arising from the fifth and sixth cervical nerves and controls abduction, extension, flexion, and rotation of the arm. This is the deltoid on the right side of the body. 